\title{
Protective Effect of Tanshinone IIA on the Early Stage of Experimental Diabetic Nephropathy
}

\author{
Su Kang KIm, ${ }^{a, \#}$ Kyung-Hee Jung, ${ }^{a, \#}$ and Byung-Cheol LeE ${ }^{*, b}$ \\ ${ }^{a}$ Kohwang Medical Research Institute, Kyung Hee University; and ${ }^{b}$ Department of Internal Medicine, College of Oriental \\ Medicine, Kyung Hee University; 1 Hoegi-dong, Dongdaemun-ku, Seoul 130-702, Korea. \\ Received August 12, 2008; accepted November 13, 2008; published online November 13, 2008
}

\begin{abstract}
Diabetic nephropathy (DN) has become the leading cause of end stage renal failure, and prevention or retardation of DN has become a major goal in biomedical research. In this study, Tanshinone IIA, a component extracted from Salvia miltiorrhiza, was studied in experimental rats in which DN was induced by streptozotocin (STZ) treatment. The DN rats were treated with $10 \mathrm{mg} / \mathrm{kg}$ of Tanshinone IIA for 12 weeks to analyze its renoprotective effect with different parameters. Renal hypertrophy and 24-h urinary protein excretion were ameliorated by Tanshinone IIA. Moreover, advanced glycation end-products (AGEs), angiotensin II (Ang II), transforming growth factor $\beta_{1}$ (TGF- $\beta_{1}$ ), collagen IV, and monocyte/macrophage (ED-1) either in the serum or kidney were significantly reduced. These results suggest that Tanshinone IIA might have protective effects on several pharmacological targets during the progression of $\mathrm{DN}$, and could be a potential drug for the prevention of DN.
\end{abstract}

Key words Tanshinone IIA; diabetic nephropathy; advanced glycation end product; angiotensin II; transforming growth factor$\beta_{1}$; collagen IV

More than $30 \%$ of diabetes mellitus patients develop clinically evident diabetic nephropathy (DN) 10 to 20 years from the onset of diabetes mellitus. DN seems to occur as a result of the interaction between metabolic and hemodynamic factors. ${ }^{1)} \mathrm{DN}$ is characterized functionally by glomerular hyperfiltration and proteinuria, and histologically by the expansion of the glomerular mesangium which is related to the loss of renal function. ${ }^{2)}$ Chronic hyperglycemia, a necessary prerequisite for the development of DN, eventually leads to the formation of long-lived non-enzymatic glycated proteins that are referred to as advanced glycation end-products (AGEs). ${ }^{3)}$ Moreover, AGEs increase extracellular matrix (ECM) production, such as collagen IV, via transforming growth factor$\beta_{1}$ (TGF- $\left.\beta_{1}\right){ }^{4}{ }^{4}$ In addition, inhibitors of angiotensin-converting enzyme (ACE), a key enzyme for hypertension control, significantly delay the onset and progression of DN. ${ }^{5)}$ Furthermore, it has been reported that ACE inhibitor (ACEi) can also inhibit the formation of AGEs, hence these inhibitors can potentially mediate diabetic complications, including nephropathy. ${ }^{\text {() }}$

Tanshinone IIA is one of the major diterpenes from Salvia miltiorrhiza (S. miltiorrhiza) referred to as 'Danshen' in traditional Chinese medicine (Fig. 1). ${ }^{7)}$ In the past, Danshen has been commonly used to treat inflammatory diseases such as edema, arthritis, hepatitis, and endangitis. ${ }^{7)}$ Danshen is also traditionally used in Asia to treat hypertension. ${ }^{8}$ Both aqueous and lipid soluble fractions of Danshen contain active components responsible for its observed clinical effects. The two active hydrophilic components of Danshen are dan-

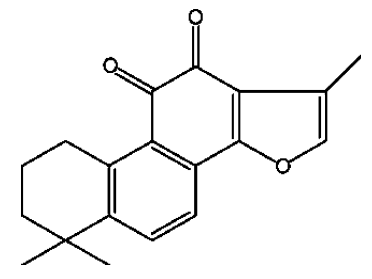

Fig. 1. Chemical Structure of Tanshinone IIA shensu and magnesium tanshinoate $\mathrm{B}$, and cryptotanshinone and Tanshinone IIA are the two lipophilic components. $\left.{ }^{8}\right)$ Tanshinone IIA has antioxidant properties and protects against lipid peroxidation in vitro and in vivo making it a potential antidote for free radical-based disorders. ${ }^{9,10)}$ In addition, it has been reported that Tanshinone IIA has neuroprotective effects during cerebral ischemia and attenuates Ang II-induced cardiac hypertrophy. ${ }^{11-13)}$ Moreover, a study by Kwark et al. showed that Tanshinone IIA may also be a therapeutic drug in the treatment of bone diseases such as osteoporosis. ${ }^{14)}$ Based on previous studies, there is a great interest in the therapeutic potential of Tanshinone IIA. To evaluate the effect of Tanshinone IIA on DN, we studied the possible influence of Tanshinone IIA on parameters such as blood glucose level, urine protein, and expression of AGEs, Ang II, TGF- $\beta_{1}$, collagen IV, and monocyte/macrophage infiltration in experimental DN rats.

\section{MATERIALS AND METHODS}

Tanshinone IIA Tanshinone IIA was purchased from the Chinese Institute for Drug and Biological Product Control (Chengdu, People's Republic of China). Tanshinone IIA was solved in $0.9 \%$ saline for oral administration.

Animals Diabetes was induced by injecting $45 \mathrm{mg} / \mathrm{kg}$ of streptozotocin (STZ) intravenously (dissolved in $\mathrm{pH} 4.5$ citrate buffer immediately before injection) into the tail vein, while controlled normal standard rats (Con group, $n=9$ ) received $2.5 \mathrm{ml} / \mathrm{kg}$ of citrate buffer. Induction of the diabetic state was confirmed by measuring blood glucose levels $72 \mathrm{~h}$ after injection of STZ. The rats whose blood glucose concentrations were $\geq 300 \mathrm{mg} / \mathrm{dl}$ were randomly divided into two groups: DN rats who received no treatment (DN group, $n=9$ ) and DN rats that were orally administrated a dose of $10 \mathrm{mg} / \mathrm{kg}$ of Tanshinone IIA daily (TS group, $n=9$ ). The animals were housed in a controlled environment $\left(24 \pm 1^{\circ} \mathrm{C}, 12-\right.$ h light: 12-h dark cycle) and were allowed food and water $\mathrm{ad}$ libitum. One day before sacrifice at 12 weeks, the animals 
were housed in metabolic cages for $24 \mathrm{~h}$ to obtain urine for the measurement of urine protein. Blood, obtained at decapitation, was collected in lithium heparin tubes, placed on ice, and serum was immediately separated and stored at $-20^{\circ} \mathrm{C}$. After animals were sacrificed, fresh kidney samples were stored in formaldehyde solution for light microscopic observation. The remaining kidneys were stored at $-75^{\circ} \mathrm{C}$ for later analysis.

Blood and Urine Analyses Plasma glucose, blood urea nitrogen (BUN), creatinine, total cholesterol, low density lipoprotein (LDL) cholesterol, triglyceride, and 24-h urinary protein excretion were analyzed by a commercial laboratory company (SMlab, Seoul, Korea).

Morphologic Analysis Kidney tissue sections $(4 \mu \mathrm{m}$ thickness) were stained with periodic acid-Schiff (PAS) and examined by light microscopy in a blinded fashion. The mesangial expansion index (MEI) was scored in four levels from 0 to 3 , with the index scores defined as follows. ${ }^{15)} 0$, normal glomeruli; 1, matrix expansion in up to $50 \%$ of glomeruli; 2, matrix expansion in $50-70 \%$ of glomeruli; 3 , matrix expansion in $75-100 \%$ of glomeruli. Scores were assigned for at least 30 glomeruli from kidney slices from each animal, and the means were calculated. To estimate MEI, the stained PAS slides were measured by two pathologists. The investigator scoring the sections was blinded to the animal group.

Immunohistochemistry Immunostaining was performed on $4 \mu \mathrm{m}$-thick sections after deparaffinization. Microwave antigen retrieval was performed in citrate buffer at $\mathrm{pH} 6.0$ for 10 min prior to peroxide quenching with $3 \% \mathrm{H}_{2} \mathrm{O}_{2}$ in phosphate buffered saline (PBS) for $10 \mathrm{~min}$. Sections were then washed in water and pre-blocked with normal goat or rabbit serum for $10 \mathrm{~min}$. For primary antibody labeling, slides were incubated with anti-AGE (Cosmo Bio, Tokyo, Japan) at a 1:500 dilution, anti-Ang II (Peninsula Laboratories, Belmont, Calif., U.S.A.) at a $1: 200$ dilution, anti-TGF- $\beta_{1}$ (Santa Cruz, CA, U.S.A.) at a $1: 200$ dilution, anti-collagen IV antibodies (SouthernBiotech, Birmingham, AL), and anti-monocyte/macrophage antigen (ED-1; Chemicon International Inc., Temecula, CA, U.S.A.) at a 1:200 dilution for $1 \mathrm{~h}$ at room temperature. The sections were then incubated with biotinylated secondary antibodies $(1: 1000)$ for $1 \mathrm{~h}$. Following a washing step with PBS, streptavidin-HRP was applied. Finally, the sections were rinsed in PBS and then developed with diaminobenzidine tetrahydrochloride substrate for $10 \mathrm{~min}$. At least three random fields of each section were examined at $\times 400$, which were analyzed by a computer image analysis system, Metavue (Molecular Devices, Sunnyvale, CA, U.S.A.). The density values represent the mean \pm S.E. of the threshold area.

Enzyme-Linked Immunosorbent Assay (ELISA) Serum samples were tested for Ang II and TGF- $\beta_{1}$ by ELISA. The plates were coated overnight with $2 \mu \mathrm{g} / \mathrm{ml}$ antiAng II and TGF- $\beta_{1}$ capture antibodies (R\&D systems, Minneapolis, MN, U.S.A.) in $0.1 \mathrm{M} \mathrm{Na}_{2} \mathrm{HPO}_{4} \mathrm{pH} 9$ buffer and blocked with PBS-Tween 20. Biotin-labeled $1 \mu \mathrm{g} / \mathrm{ml}$ antiAng II and TGF- $\beta_{1}$ detecting antibodies were used. The plates were developed using streptavidin-horseradish peroxidase (Vector, Burlingame, CA, U.S.A.) and 2,2-azino-bis substrate (Sigma, St. Louis, MO, U.S.A.).

Statistical Analyses All of the values are expressed as the mean \pm S.E. Significant differences between the groups were analyzed using a one-way analysis of variance (ANOVA), followed by a non-parametric post hoc test (LSD). A $p$-value of 0.05 or less was considered statistically significant.

\section{RESULTS}

Functional and Biochemical Parameters The DN group demonstrated significantly higher plasma glucose than the control group $(p<0.001)$ (Table 1). Plasma glucose decreased in Tanshinone IIA treatment group compared to DN group $(p<0.05)$. Furthermore, BUN and $24-\mathrm{h}$ urine protein excretion in DN rats were all markedly elevated compared with controls $(p<0.01)$. As expected, Tanshinone IIA treatment significantly decreased BUN and 24-h urine protein excretion levels $(p<0.05)$.

Kidney Histopathology Structural abnormalities of DN are similar in type 1 and type 2 diabetes, ${ }^{16)}$ and all renal compartments (glomeruli, tubulointerstitium, and renal vasculature) can be affected. The morphological change of DN is mesangial expansion due to increased mesangial matrix deposition, a mild increase in mesangial cellularity, and hypertrophy of mesangial cells. ${ }^{17)}$ In this study, kidney sections were stained with PAS for histochemical determination of mucopolysaccharides. PAS reaction was detected in some glomeruli from diabetic animals. Also, mesangial matrix expansion was observed in DN groups (Fig. 2). However, in the Tanshinone IIA treatment group, the PAS reaction was slightly less than in the DN group. In addition, the Tanshinone IIA treatment partially prevented mesangial matrix expansion. The MEI in PAS-stained glomeruli was determined in DN rats. The MEI in the DN group (1.7 \pm 0.2$)$ was remarkably increased compared with the control group $(p<0.01$, $0.3 \pm 0.1)$, whereas the MEI in the Tanshinone IIA treatment group $(0.9 \pm 0.1)$ was significantly lower than that of the DN group $(p<0.05)$.

The Protein Expression of AGEs, Ang II TGF- $\beta_{1}$, Collagen IV and ED-1 We next assessed the effects of Tanshinone IIA on the expression level of AGEs, Ang II, TGF- $\beta_{1}$, collagen IV, and ED-1 by immunohistochemistry. The expression of AGEs in DN (30.51 \pm 1.62$)$ was significantly increased compared with the controls $(p<0.05,10.68 \pm 1.74)$

Table 1. The Effect of Tanshinone IIA Treatment on Biochemical Parameters of Diabetic Nephropathy (DN) Rats

\begin{tabular}{|c|c|c|c|}
\hline \multirow{2}{*}{ Parameter } & \multicolumn{3}{|c|}{ Group } \\
\hline & Con & $\mathrm{DN}$ & TS \\
\hline Body weight (g) & $457.6 \pm 24.84$ & $347.3 \pm 13.18^{\#}$ & $348.4 \pm 43.68$ \\
\hline Plasma glucose (mg/dl) & $140.8 \pm 11.56$ & $608.2 \pm 33.60^{\# \#}$ & $530.0 \pm 72.15^{*}$ \\
\hline Blood urea nitrogen $(\mathrm{mg} / \mathrm{dl})$ & $19.8 \pm 0.45$ & $28.1 \pm 1.32^{\# \#}$ & $22.0 \pm 2.27 *$ \\
\hline Creatinine $(\mathrm{mg} / \mathrm{dl})$ & $0.53 \pm 0.01$ & $0.55 \pm 0.01$ & $0.57 \pm 0.01$ \\
\hline $\operatorname{Albumin}(\mathrm{mg} / \mathrm{dl})$ & $2.33 \pm 0.04$ & $2.04 \pm 0.07$ & $2.06 \pm 0.13$ \\
\hline Total cholesterol (mg/dl) & $76.80 \pm 4.84$ & $96.00 \pm 5.67$ & $76.60 \pm 4.49$ \\
\hline LDL cholesterol (mg/dl) & $8.00 \pm 0.84$ & $15.71 \pm 2.97$ & $13.20 \pm 1.16$ \\
\hline Triglyceride (mg/dl) & $128.60 \pm 15.05$ & $160.71 \pm 35.97$ & $178.40 \pm 23.88$ \\
\hline $\begin{array}{l}\text { Urinary protein excretion } \\
(\mathrm{mg} / \mathrm{kg} / 24 \mathrm{~h})\end{array}$ & $22.3 \pm 5.8$ & $174.6 \pm 15.3^{\# \#}$ & $77.7 \pm 18.2 *$ \\
\hline
\end{tabular}

Data are presented as mean \pm S.E. $(n=9)$. \# $p<0.05$, \# $p<0.01$, \#\# $p<0.001 v$ s. Con and $* p<0.05 v s$. DN, respectively. Con, control; DN, diabetic nephropathy $(45 \mathrm{mg} / \mathrm{kg}$ of STZ); TS, diabetic rats treated with Tanshinone IIA $(10 \mathrm{mg} / \mathrm{kg})$. 
The increased expression of AGEs observed in glomeruli was reduced by treatment with Tanshinone IIA $(p<0.05$, $20.26 \pm 1.02)$. Furthermore, the expression of Ang II in the DN group (24.64 \pm 0.53$)$ was decreased by Tanshinone IIA $(p<0.05,15.06 \pm 0.75)$. Like Ang II, expression of TGF- $\beta_{1}$ in the DN group $(29.79 \pm 2.11)$ was significantly higher than in the control group $(8.53 \pm 1.01)$, and was located mainly in the glomeruli $(p<0.01)$, suggesting that TGF- $\beta_{1}$ expression is correlated with ECM accumulation in glomeruli. However, Tanshinone IIA treatment prevented the enhanced expression of TGF- $\beta_{1}(16.25 \pm 2.21)$. Faint collagen IV expression was observed in normal glomeruli, mainly in the glomerular basement membrane (Fig. 3). In the DN group, expression of collagen IV (30.52 \pm 1.79$)$ was significantly increased compared with the control group $(p<0.01,6.69 \pm 0.82)$, whereas
Tanshinone IIA treatment partially attenuated increased collagen IV expression $(p<0.01,14.26 \pm 3.17)$. The expression of ED-1 in DN (11.71 \pm 0.05$)$ was significantly increased compared with the controls $(p<0.05,0.27 \pm 0.20)$. The increased expression of ED-1 observed in glomeruli was reduced by treatment with Tanshinone IIA $(p<0.05,4.45 \pm$ 1.89).

The Production of Ang II and TGF- $\boldsymbol{\beta}_{1}$ As shown in Fig. 4, the level of Ang II in serum of the DN group was significantly higher than that of the control groups $(p<0.01$, $2.84 \pm 0.47 \mathrm{ng} / \mathrm{ml}$ ). Ang II levels in serum in the Tanshinone IIA treatment group was reduced significantly compared to the DN group $(p<0.05,1.79 \pm 0.03 \mathrm{ng} / \mathrm{ml})$. Furthermore, in the DN group, TGF- $\beta_{1}$ levels in serum $(153.4 \pm 22.02 \mathrm{pg} / \mathrm{ml})$ was similar to that of Ang II; TGF- $\beta_{1}$ levels in serum, how-
A

Con

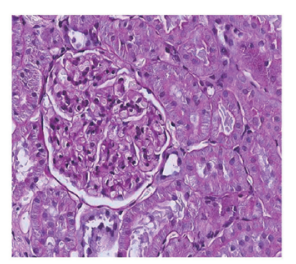

DN

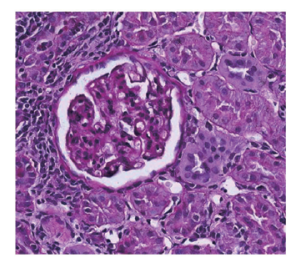

TS

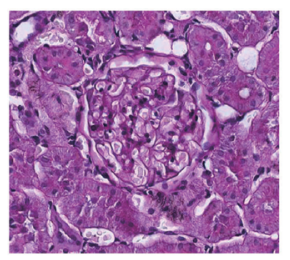

B

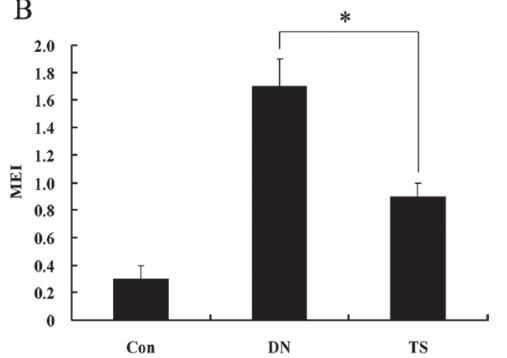

Fig. 2. Histological Analysis of Diabetic Nephropathy (DN) Rats by Periodic Acid Schiff (PAS) Staining

(A) Kidney sections were stained with PAS in streptozotocin (STZ)-induced diabetic nephropathy. (B) Mesangial expansion index (MEI) in PAS-stained glomeruli was scored according to four levels, and the average value for each rat was obtained from the analysis of $>30$ glomeruli per rat. Data are presented as the mean \pm S.E. $(n=9)$. $* p<0.05 v s$. DN. Con, control; TS, diabetic rats treated with Tanshinone IIA $(10 \mathrm{mg} / \mathrm{kg})$. Original magnification $\times 400$.

A

AGE

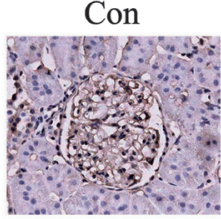

Ang II

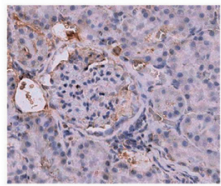

TGF- $\beta_{1}$

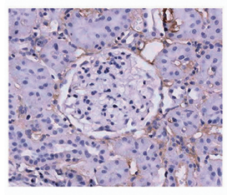

Collagen IV

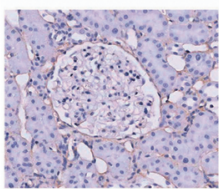

ED-1

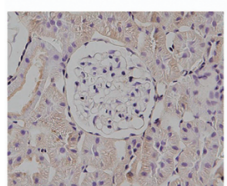

$\mathrm{DN}$
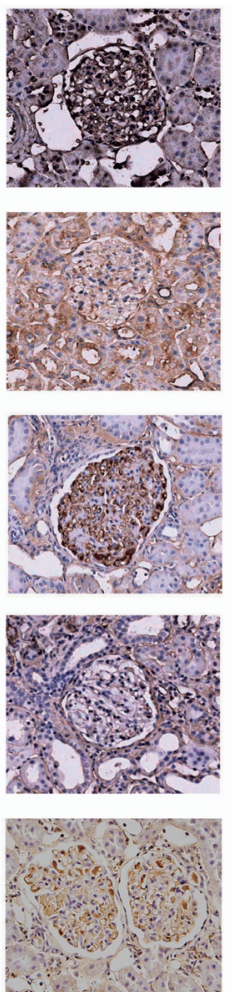

TS
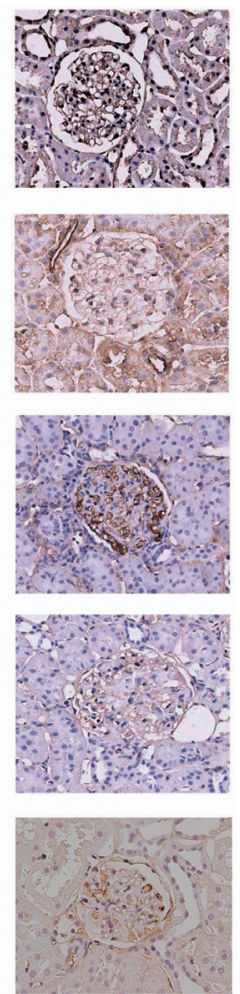

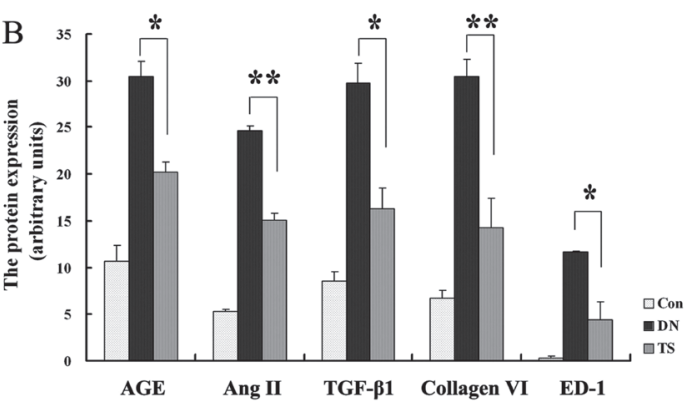

Fig. 3. The Expression of Advanced Glycation End-Products (AGEs), Angiotensin II (Ang II), Transforming Growth Factor- $\beta_{1}$ (TGF- $\beta_{1}$ ), Collagen Type IV, and ED-1 after Treatment with Tanshinone IIA

(A) Kidney tissues were stained with anti-AGEs, anti-Ang II, anti-TGF- $\beta_{1}$, collagen IV, and ED-1 antibodies. (B) Quantification of AGEs, Ang II, TGF- $\beta_{1}$, Collagen IV, and ED1 expression was performed using Metavue software. Data are presented as the mean \pm S.E. of at least six separate experiments. $* p<0.05, * * p<0.01, v s$. DN, respectively. Con, control; DN, diabetic nephropathy (45 mg/kg of STZ); TS, diabetic rats treated with Tanshinone IIA (10 mg/kg). Original magnification $\times 400$. 

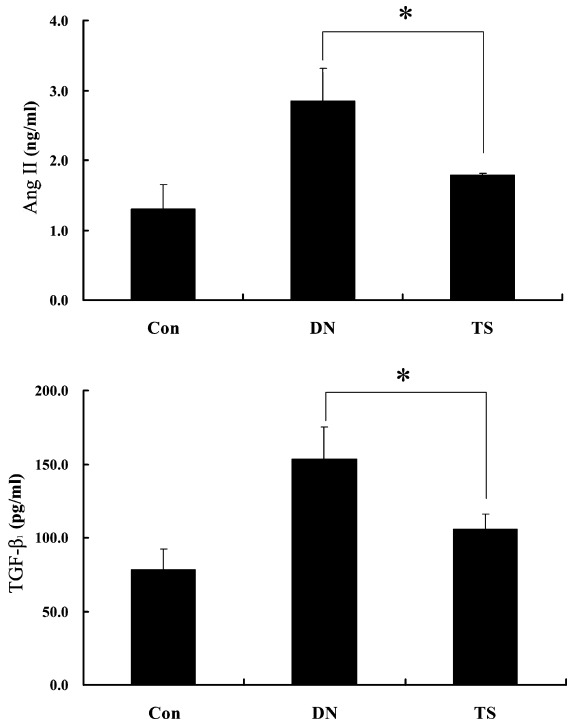

Fig. 4. ELISA Measurements of the Levels of Angiotensin II (Ang II) and Transforming Growth Factor- $\beta_{1}\left(\right.$ TGF- $\left.\beta_{1}\right)$ in Serum after Treatment with Tanshinone IIA

After treatment with Tanshinone IIA, rats in each group were killed and venous blood was collected. Serum samples were bound with anti-Ang II and anti-TGF- $\beta_{1}$ antibodies. Data are presented as mean \pm S.E. for at least six separate experiments. $* p<0.05$ vs. DN. Con, control; DN, diabetic nephropathy (45 mg/kg of STZ); TS, diabetic rats treated with Tanshinone IIA $(10 \mathrm{mg} / \mathrm{kg})$.

ever, was decreased significantly by Tanshinone IIA treatment $(p<0.05,105.92 \pm 10.21 \mathrm{pg} / \mathrm{ml})$.

\section{DISCUSSION}

Multiple mechanisms have been postulated for the development of diabetic nephropathy, including AGEs, type IV collagen, TGF- $\beta$, polyol pathway, oxidative stress, inflammation, and hemodynamic changes. Therefore, multi-targeted therapeutic interventions should be required for DN. Recently, it has been reported that several herbs such as $\mathrm{Mo}$ mordica grosvenori and Erigeron breviscapus, have multitargeted reno-protective effect in $\mathrm{DN} .^{18,19)}$ In the present study, we demonstrated the multi-targeted renoprotective effects of Tanshinone IIA on DN. To the best of our knowledge, this is the first report showing that Tanshinone IIA treatment improves DN status according to plasma glucose, BUN, and urine protein excretion. Furthermore, our data indicates that Tanshinone IIA can prevent streptozotocin-induced DN by deceasing the expression of AGEs, Ang II, TGF- $\beta_{1}$, collagen IV, and ED-1. Also, histological examination revealed that Tanshinone IIA significantly ameliorated diabetic-induced mesangial expansion.

$\mathrm{DN}$ is one of the main complications of diabetes, and involves increased urine protein excretion and loss of renal function. Thus, increased urine protein is a key component of DN. Many studies have shown correlations between urine protein level and a decline in glomerular filtration rate. ${ }^{20)}$ Indeed, our study showed that Tanshinone IIA decreased urine protein excretion by $60 \%$ in STZ-induced DN, which was similar to anti-proteinuric effects of ACEi or Ang II receptor blocker (ARB) in previous reported studies. ${ }^{21,22)}$

AGEs accumulate in diabetic tissue at an accelerated rate as a consequence of increased glucose concentration in extra- cellular fluids. The administration of AGEs to non-diabetic animals leads to glomerulosclerosis and albuminuria, ${ }^{23)}$ suggesting that AGEs alone may be sufficient to induce renal injury in diabetes patients. Recently, it has been reported that AGEs stimulate Ang II production by an interaction with receptor for AGEs. ${ }^{24}$ ) Ang II, the primary effector product of the renin-angiotensin system, is thought to be a crucial factor in progressive renal failure. ${ }^{25)}$ In fact, ARBs have shown a blocking effect on the progression of albuminuria, nephropathy development, and decline in kidney function. ${ }^{26-28)}$ This study showed that expression of AGEs in the kidney of DN rats was decreased by Tanshinone IIA. Moreover, Tanshinone IIA decreased Ang II production and urine protein excretion in $\mathrm{DN}$.

TGF- $\beta_{1}$ is reported to mediate renal hypertrophy, glomerulosclerosis, and tubulointerstitial fibrosis in DN. Many features of the diabetic state stimulate renal TGF- $\beta_{1}$ activity in both cell culture and in vivo systems. Once activated, TGF- $\beta_{1}$ induces the accumulation of matrix proteins ${ }^{29)}$ and stimulates the mRNA expression and production of key ECM molecules such as collagen $\mathrm{IV}^{29,30}$ ) There are some reports indicating that inhibition of TGF- $\beta_{1}$ significantly decreases the high glucose-induced increase in ECM protein expression. ${ }^{31)}$ In the present study, increased TGF- $\beta_{1}$ in DN was markedly inhibited by Tanshinone IIA. The expression of collagen IV was also increased in DN as expected from the increased TGF- $\beta_{1}$ levels, and Tanshinone IIA inhibited the expression of collagen IV consistent with its inhibition of TGF- $\beta_{1}$ expression.

Many studies have reported that Tanshinone IIA has the capacity of anti-inflammation. ${ }^{5,32)}$ Fang et al. reported that Tanshinone IIA not only inhibits the oxidation but also suppresses the inflammation in atherosclerosis that is a chronic inflammatory disease. ${ }^{33)}$ We evaluated the effect of Tanshinone IIA on glomerular infiltration of monocyte/macrophages. ED-1 was quantified because they play a major role in early healing and reflect the inflammatory healing process. ${ }^{34)}$ In the present study, increased ED-1 in DN was markedly decreased in Tanshinone IIA treatment.

In summary, Tanshinone IIA treatment in this study decreased glucose, BUN, and 24-h urine protein excretion in a STZ-induced DN rat model. Moreover, Tanshinone IIA significantly inhibited the expressions of AGEs, Ang II, TGF$\beta_{1}$, collagen IV, and ED-1 in this model. Tanshinone IIA has protective effects on several pharmacological targets in DN. Therefore, we suggest that Tanshinone IIA might be useful in preventing the development of DN. In the future studies, Tanshinone IIA therapy should be compared to combined ACEi or ARB to establish the benefit of this treatment, as well as to define its optimal dose and clear its primary action mechanism on DN.

Acknowledgements This research was supported by the Program of Kyung Hee University for the Young Researcher of Medical Science in 2007 (KHU-20071472).

\section{REFERENCES}

1) Cooper M. E., Gilbert R. E., Epstein M., Metabolism, 47, 3-6 (1998).

2) Mauer S. M., Steffes M. W., Ellis E. N., Sutherland D. E., Brown D. M., Goetz F. C., J. Clin. Invest., 74, 1143-1155 (1984).

3) Monnier V. M., Sell D. R., Nagaraj R. H., Miyata S., Grandhee S., 
Odetti P., Ibrahim S. A., Diabetes, 41 (Suppl. 2), 36-41 (1992).

4) Doi T., Vlassara H., Kirstein M., Yamada Y., Striker G. E., Striker L. J., Proc. Natl. Acad. Sci. U.S.A., 89, 2873-2877 (1992).

5) Throckmorton D. C., Brogden A. P., Min B., Rasmussen H., Kashgarian M., Kidney Int., 48, 111-117 (1995).

6) Miyata T., van Ypersele de Strihou C., Ueda Y., Ichimori K., Inagi R., Onogi H., Ishikawa N., Nangaku M., Kurokawa K., J. Am. Soc. Nephrol., 13, 2478-2487 (2002).

7) Jang S. I., Jeong S. I., Kim K. J., Kim H. J., Yu H. H., Park R., Kim H. M., You Y. O., Planta Med., 69, 1057-1059 (2003).

8) Zhou L., Zuo Z., Chow M. S., J. Clin. Pharmacol., 45, 1345-1359 (2005).

9) Niu X. L., Ichimori K., Yang X., Hirota Y., Hoshiai K., Li M., Nakazawa H., Free Radic. Res., 33, 305-312 (2000).

10) Wang A. M., Sha S. H., Lesniak W., Schacht J., Antimicrob. Agents Chemother, 47, 1836-1841 (2003).

11) Takahashi K., Ouyang X., Komatsu K., Nakamura N., Hattori M., Baba A., Azuma J., Biochem. Pharmacol., 64, 745-749 (2002).

12) Xia W. J., Yang M., Fok T. F., Li K., Chan W. Y., Ng P. C., Ng H. K., Chik K. W., Wang C. C., Gu G. J., Woo K. S., Fung K. P., Pediatr. Res., 58, 784-790 (2005).

13) Lam B. Y., Lo A. C., Sun X., Luo H. W., Chung S. K., Sucher N. J., Phytomedicine, 10, 286-291 (2003).

14) Kwak H. B., Yang D., Ha H., Lee J. H., Kim H. N., Woo E. R., Lee S., Kim H. H., Lee Z. H., Exp. Mol. Med., 38, 256-264 (2006).

15) Sugaru E., Sakai M., Horigome K., Tokunaga T., Kitoh M., Hume W. E., Nagata R., Nakagawa T., Taiji M., Am. J. Physiol. Renal. Physiol., 289, F998-F1004 (2005).

16) Parving H. H., Kidney Int., 60, 2041-2055 (2001).

17) Wolf G., Reinking R., Zahner G., Stahl R. A., Shankland S. J., Diabetologia, 46, 1090-1099 (2003).
18) Song F., Qi X., Chen W., Jia W., Yao P., Nussler A. K., Sun X., Liu L., Eur. J. Nutr., 46, 61-69 (2007).

19) Qi X. M., Wu G. Z., Wu Y. G., Lin H., Shen J. J., Lin S. Y., Nephron Exp. Nephrol., 104, e147-157 (2006).

20) Mogensen C. E., N. Engl. J. Med., 310, 356-360 (1984).

21) Zhang Y., Chen B., Hou X. H., Guan G. J., Liu G., Liu H. Y., Li X. G., Chin. Med. J. (Engl.), 120, 988-995 (2007).

22) Allard J., Buleon M., Cellier E., Renaud I., Pecher C., Praddaude F., Conti M., Tack I., Girolami J. P., Am. J. Physiol. Renal Physiol., 293, F1083-F1092 (2007).

23) Vlassara H., Striker L. J., Teichberg S., Fuh H., Li Y. M., Steffes M., Proc. Natl. Acad. Sci. U.S.A., 91, 11704-11708 (1994).

24) Fukami K., Ueda S., Yamagishi S., Kato S., Inagaki Y., Takeuchi M., Motomiya Y., Bucala R., Iida S., Tamaki K., Imaizumi T., Cooper M. E., Okuda S., Kidney Int., 66, 2137-2147 (2004).

25) Kagami S., Border W. A., Miller D. E., Noble N. A., J. Clin. Invest., 93, 2431-2437 (1994).

26) Mathiesen E. R., Hommel E., Giese J., Parving H. H., BMJ, 303, 8187 (1991).

27) ACE Inhibitors in Diabetic Nephropathy Trialist Group, Ann. Intern. Med., 134, 370-379 (2001).

28) The EUCLID Study Group, Lancet, 349, 1787-1792 (1997).

29) Chen S., Jim B., Ziyadeh F. N., Semin. Nephrol., 23, 532-543 (2003).

30) Sharma K., Ziyadeh F. N., Semin. Nephrol., 17, 80-92 (1997).

31) Ha H., Yu M. R., Lee H. B., Kidney Int., 59, $463-470$ (2001).

32) Zhan C., Tao X., Tian C., Xiong W., Zheng Z., Chinese Journal of Clinical Rehabilitation, 9, 254-256 (2005).

33) Fang Z. Y., Lin R., Yuan B. X., Yang G. D., Liu Y., Zhang H., J. Ethnopharmacol., 115, 217-222 (2008).

34) Duffield J. S., Clin. Sci. (London), 104, 27-38 (2003). 